\title{
Validation of the Image Registration Technique from Functional Near Infrared Spectroscopy (fNIRS) Signal and Positron Emission Tomography (PET) Image
}

\author{
Fairuz Mohd Nasir, Hiroshi Watabe
}

\begin{abstract}
Functional near infrared spectroscopy (fNIRS) is an imaging system that can measure hemodynamic changes of the brain. However, the system incapability to measure beyond the brain cortex region make it usage less appealing for in-depth brain studies. To overcome this, many researchers combine fNIRS with other imaging modalities to gain better understanding of the brain activities. In this paper, we described the theory of the registering fNIRS signals and positron emission tomography (PET) image method and performed experiments to validate it. The registration method was validated using specially designed phantom for fNIRS and PET. Polaris system was used to track the position of the phantom which is based on the Polaris markers during INIRS and PET procedures. The Polaris markers share the same coordinate, thus the fNIRS and PET were calibrated to each other through these markers. To register the fNIRS signal on the PET image, the phantom position in fNIRS coordinate is translated to PET coordinate which allow the probe and the markers being coordinated in PET. Polaris markers were used as the references marker to determine the transformation matrices. The result shows that the fNIRS channel can be viewed on the PET image of the phantom. The transformation error from Polaris to PET is less than $1.00 \mathrm{~mm}$ and the precision test is less than $0.1 \mathrm{~mm}$ while the accuracy is less than $2.8 \mathrm{~mm}$. This result suggests that our theory on the registration method could be used for multimodal image registration between fNIRS and other modalities.
\end{abstract}

Keywords: functional near-infrared spectroscopy, PET-fNIRS, multimodal image registration

\section{INTRODUCTION}

$\mathrm{M}_{\mathrm{u}}$ ultimodal images are gaining attention among medical practitioners in enhancing their diagnosis the medical images. To produce a good multimodal image, one important criterion that needs to be considered is the registration of the multimodal images. According to Calvin Maurer 2004, image registration could be defined as one-to-one mapping between two coordinates in different spaces where the two

Revised Manuscript Received on July 22, 2019.

* Correspondence Author

Fairuz Mohd Nasir*, University Sultan Zainal Abidin, Faculty of Health Sciences, Campus Gong Badak, 21300 Kuala Terengganu, Malaysia. Email: fairuz.unisza@gmail.com

Hiroshi Watabe, Divisions of Radiation Protection and Safety Control, Cyclotron and Radioisotope Center, Tohoku University, 6-3 Aoba, Aramaki, Aoba-ku, Sendai, Miyagi 980-8578, Japan Email: watabe@cyric.tohoku.ac.jp

(C) The Authors. Published by Blue Eyes Intelligence Engineering and Sciences Publication (BEIESP). This is an open access article under the CC BY-NC-ND license (http://creativecommons.org/licenses/by-nc-nd/4.0/) coordinates correspond to the same anatomical point to each other [1]. Meanwhile, multimodal image registration is matching images of the same patient which acquired using different imaging modalities. Currently, there are a lot of medical imaging modalities such as computed tomography (CT), magnetic resonance imaging (MRI), single-photon emission computed tomography (SPECT) and positron emission tomography (PET) which have their specialties and drawbacks. Primary concern of most researcher in optimizing the advantages and specialties of these imaging modalities, is how to register images originated from the same patient but different modalities while expanding the anatomical and physiological information obtained.

Most of the existed integrated system such as PET-CT and PET-MRI does not need image registration as they acquired the image simultaneously and share the same coordinate. Although they are separate modalities, image fusion is being done by registering the images together. But this integrated system has it owns disadvantages due to the high installation cost and hardware restriction. There are two methods of image registrations; Software-based registration such as SPM (statistical parametric mapping) and FSL which was developed in Oxford University, UK. The other method of registration is hardware-based registration where it uses an external device to determine the transformation function. Commonly used registering method is software-based registration as it only needs a computer. However, the result of this method is highly dependent on the image quality, and the chosen algorithm. On the other hand, hardware-based registration is fast and accurate, but it needs specific hardware while motion and non-rigid deformation may cause signal not able to register internal structure correctly. In this paper, we focus on the multimodal registration of functional near-infrared spectroscopy (fNIRS) and PET signal. fNIRS is an imaging technique that use near-infrared light to measure oxygen concentration changes in blood hemoglobin. The changes in oxygen concentration are being presented in two-dimension (2D) signals and the interpretation of the oxygen level is according to the area of the measurement channel. fNIRS is advantageous in measuring cerebral hemodynamic during brain activation as it has good temporal resolution, but it only capable of measuring the oxygen changes in the cortex region.

Published By:

Blue Eyes Intelligence Engineering

\& Sciences Publication

(C) Copyright: All rights reserved.

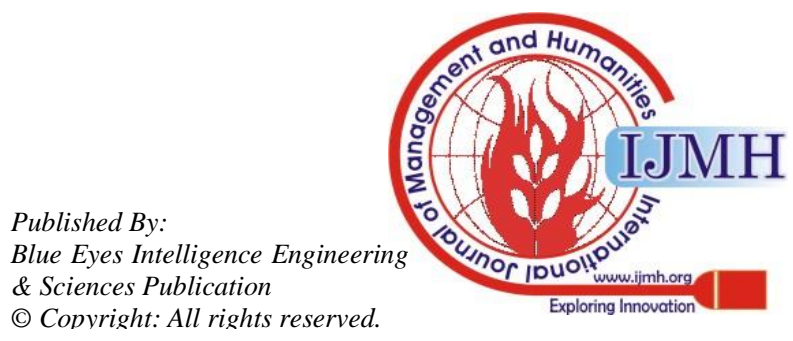




\section{Validation of the Image Registration Technique from Functional Near Infrared Spectroscopy (fNIRS) Signal and Positron Emission Tomography (PET) Image}

Thus, many researchers combined or compared the fNIRS information with others imaging modalities. The fNIRS result is being frequently compares with blood-oxygen-level-dependent (BOLD) fMRI as the BOLD response in fMRI is related to deoxyhemoglobin [2][3-8]. Moreover, fNIRS monitoring were often used alongside PET scan to study the cerebral oxygen and cerebral blood flow during brain activation $[9,10]$. Thus, it would be very beneficial if fNIRS signals can be view on another medical image. However, first, fNIRS signal registration with other modalities need to be established.

This paper will review the fNIRS and PET registration theory and its validation method. As the registration method of fNIRS signal and PET image has already been performed on human and published [11], this paper will only describe the signal registration method theory and validation using specially designed phantom for fNIRS and PET.

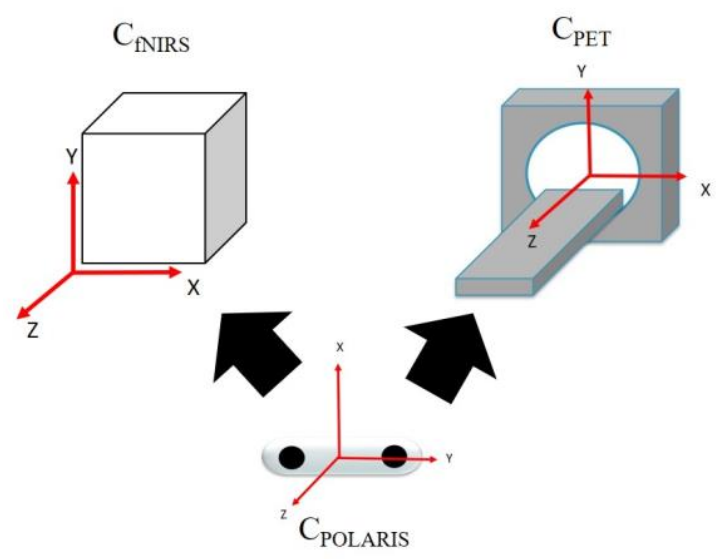

Fig. 1. The schematic diagram of the coordinate system in the registration. $\mathrm{C}_{\mathrm{fNIRS}}$ represent the fNIRS coordinate of the probes position during fNIRS measurement, $\mathrm{C}_{\mathrm{PET}}$ represent the coordinate system of the PET image whearas CPOLARIS represent coordinate system tracked by Polaris.

\section{THEORY OF THE REGISTRATION METHOD}

Fig. 1 showed the schematic diagrams and coordinates of the registration method. In this method, the coordinates of the fNIRS signal probe are translated from fNIRS coordinate ( $\mathrm{C}_{\mathrm{fNIRS}}$ to PET coordinate $\left(\mathrm{C}_{\mathrm{PET}}\right.$ ) based on the references marker given by Polaris coordinate $\left(\mathrm{C}_{\mathrm{Pol}}\right)$. Polaris is an optical tracking system that detects the position and orientation of Polaris markers within a specific measurement volume. Polaris provides precise, real-time spatial measurements of the location of the marker and can track multiple markers simultaneously. In this study, Polaris system used to track the three-dimensional (3D) position of Polaris markers during fNIRS and PET measurement procedure. These $3 \mathrm{D}$ coordinate act as a reference point to translate the fNIRS probe to the PET coordinate, as they share the same coordinate.

fNIRS is the measurement using emitting and receiving probe whereas fNIRS signals are measured in the channels in between those two probes. In this study, we used specially designed fNIRS-PET phantom to validate the registration method. The position of the fNIRS channels and the probes are illustrated in fig. 2. The red color blocks indicate the transmitting probes and the blue color blocks are the receiving probes whereas the numbers in the yellow color blocks indicate the channel number.

To view the fNIRS signals on the PET image, these channels must be registered on the PET image, thus the probes information must be translated from $\mathrm{C}_{\text {fNIRS }}$ to $\mathrm{C}_{\mathrm{PET}}$ as shown in fig. 3. Using information of these positions and rigid body transformation, the acquired signal was registered to the $\mathrm{C}_{\mathrm{PET}}$ based on the reference marker which is being shared between the two modalities.

\section{VALIDATION EXPERIMENT}

This experiment is carried out to validate the registration method. In this experiment, we determined the position of the fNIRS probe in $\mathrm{C}_{\mathrm{PET}}$ and register on the PET image and test the precision and accuracy of the translation process from $\mathrm{C}_{\mathrm{fNIRS}}$ to $\mathrm{C}_{\mathrm{PET}}$.

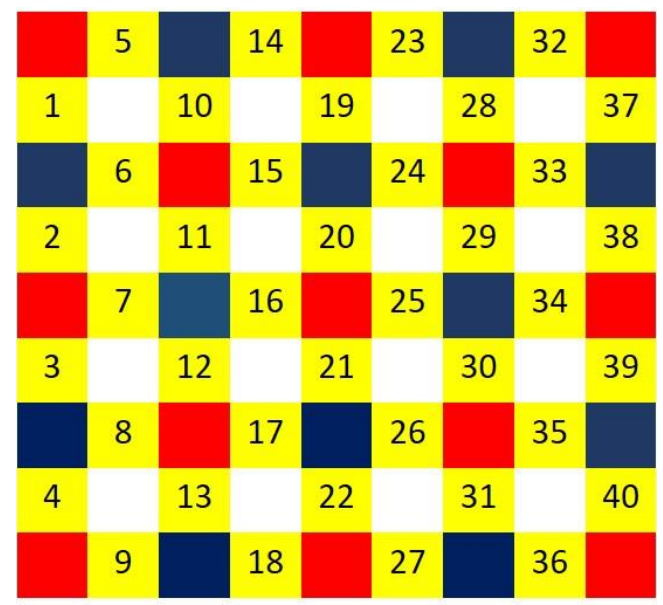

Fig. 2. The illustration of the emitting, receiving and the measurement signal channels. The red and blue color blocks are transmitting and receiving probe respectively whereas the numbers in the yellow color blocks are the channel number.

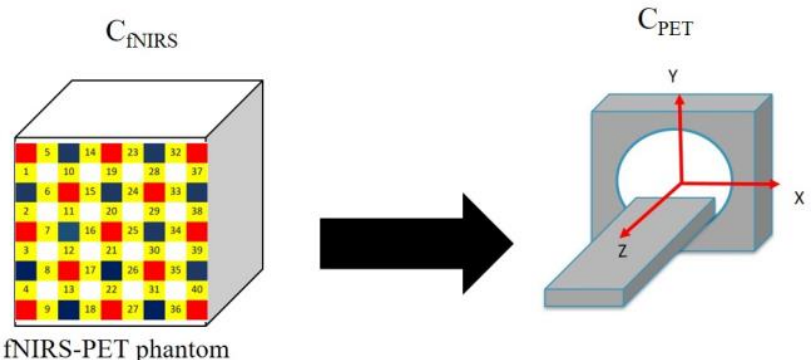

Fig. 3. The illustration of the transformation of the phantom from $\mathrm{C}_{\mathrm{fNIRS}}$ to the $\mathrm{C}_{\text {PET }}$. The coordinate of the probe on the phantom must be transformed to the PET coordinate in order to register the probe on the PET image.

\section{A. fNIRS-PET Phantom Set Up}

This experiment was performed to validate the phantom which is being specially designed to be measured by fNIRS and PET. The phantom was set up as in fig. 4 to acquire fNIRS signal. The phantom was filled up with aqueous lipid solution, and the fNIRS probe was fixed from the bottom of the phantom tank.

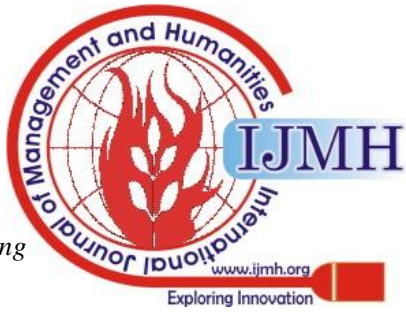


The source holder put in place the black ink or radioactive source when the aqueous lipid solution was being filled in the phantom tank. The black ink was used in the fNIRS measurement while the Na-22-point source was used for PET scanning. Four Polaris markers were placed on the phantom that will be detected by Polaris system during the fNIRS examination and PET scan. Because the fNIRS probes were fixed from the bottom of the phantom, one sheet with the exact same position as the probe was made and placed on the side of the phantom which facing the Polaris. Four Polaris markers were placed in between the probe position randomly.

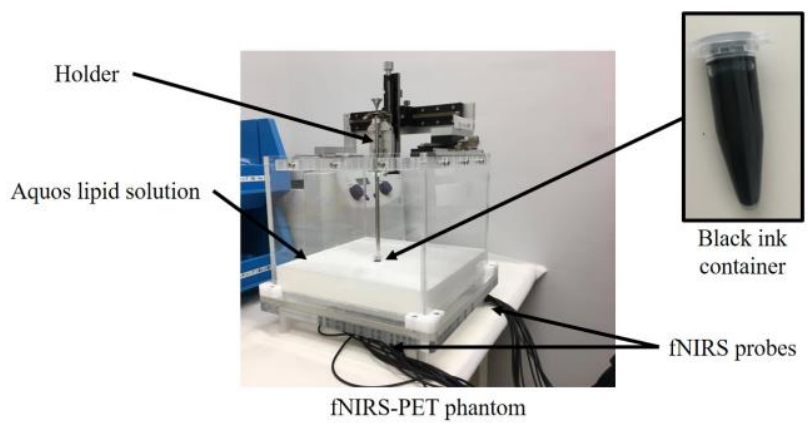

Figure 4: The fNIRS-PET phantom setup.

\section{B. fNIRS Measurement}

Fig. 5 shows the position of Polaris and fNIRS-PET phantom position during fNIRS measurement. The fNIRS signal was recorded as the container holder is moved toward the bottom on channel 6. During the same time, Polaris system tracked the position of Polaris markers on the phantom.

In order to test the Polaris marker transformation precision from different phantom positions, this measurement was repeated using two other positions where; first, the phantom was moved $5 \mathrm{~cm}$ to the right and second, $5 \mathrm{~cm}$ towards the Polaris.

\section{PET Scanning}

After the fNIRS measurement, the phantom was brought to the PET room and positioned on the PET bed. The Polaris markers side were placed facing the Polaris as shown in fig. 6 . The black ink container was replaced with the Na-22-point source and positioned the holder on top of channel 6. During the PET scanning, Polaris system read the position of the markers.

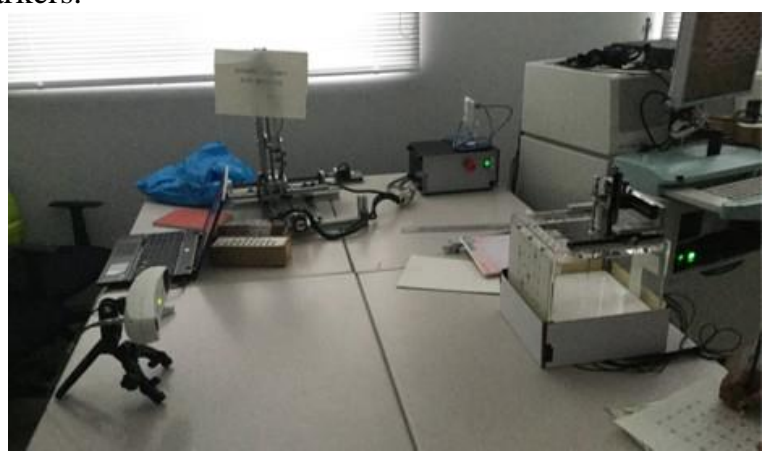

Fig. 5: The position of the Polaris and phantom during the fNIRS measurement.

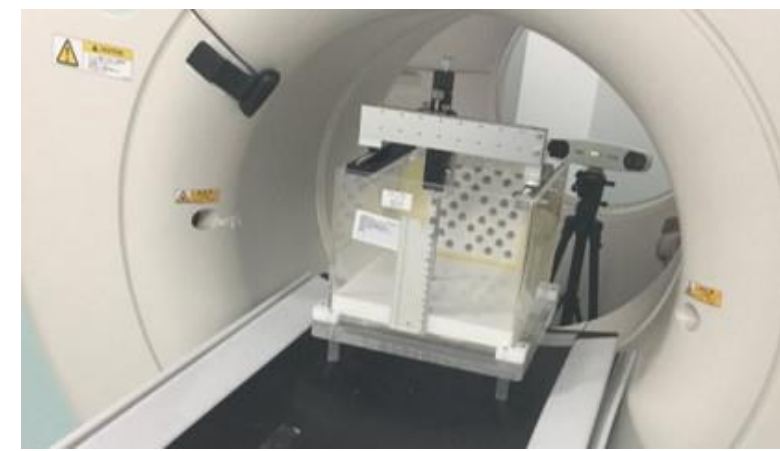

Fig. 6. The position of the phantom and Polaris during PET scan.
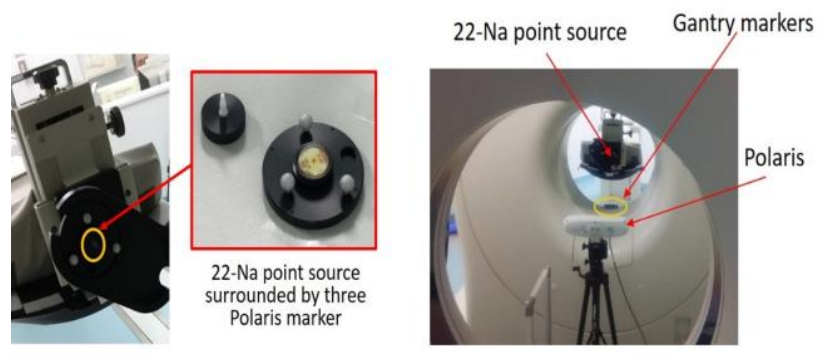

Fig. 7. a) The Na-22-point source which surrounded by three Polaris markers, and placed on the adjustable tool and b) the set-up of the calibration experiment where Polaris read the markers around the source and the gantry markers.

\section{Calibration from Polaris to PET coordinate}

The calibration study was conducted to determine the transformation matrixes from $\mathrm{C}_{\text {Pol }}$ to $\mathrm{C}_{\text {Рет. }}$. The calibration experiment was carried out using $\mathrm{Na}$-22-point source which is mounted on the adjustable tool as shown in fig. 7a. There are three Polaris markers surrounded the source. Six PET scans were performed on the source and for each scan, the source was moved to a different position by moving the source holder of the adjustable tool. During each scan, the Polaris read the markers and the gantry markers. Fig. 7b shows the position of Polaris and the adjustable tool during the experiment. Both of source coordinate given by Polaris and PET were relatively corresponds to each other. Using the position of markers in both $\mathrm{C}_{\mathrm{Pol}}$ and $\mathrm{C}_{\mathrm{PET}}$, the transformation matrix from Polaris to PET was determined.

\section{E. Transformation}

The transformation is performed to bring the probe coordinate and marker coordinate from fNIRS to PET. The following are the transformation steps to get the probe and marker coordinate in $\mathrm{C}_{\mathrm{PET}}$.

The marker coordinate, $\mathrm{M}_{\mathrm{fNIRS}}(\mathrm{x}, \mathrm{y}, \mathrm{z})$ in $\mathrm{C}_{\mathrm{fNIRS}}$ is corresponded to the marker location, $\mathrm{M}_{\text {Pol_1 }}(\mathrm{x}, \mathrm{y}, \mathrm{z})$ in $\mathrm{C}_{\text {Pol_1 } 1 \text {, }}$ as shows in the following relationship;

$$
\mathrm{M}_{\text {Pol_1 }}=\mathrm{T}_{\mathrm{fNIRS} \rightarrow \mathrm{Pol} \_1} * \mathrm{M}_{\mathrm{fNIRS}}
$$

Therefore, $\mathrm{T}_{\mathrm{fNIRS}} \rightarrow \mathrm{Pol}_{-} 1$ is given by the following relationship;

$$
\mathrm{T}_{\mathrm{fNIRS} \rightarrow \text { Pol } \_1}=\mathrm{M}_{\mathrm{Pol} \_1} * \mathrm{M}_{\mathrm{fNIRS}}{ }^{-1}
$$

Published By:

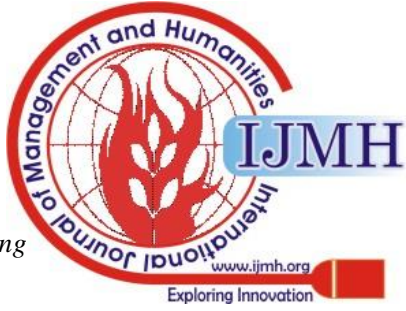




\section{Validation of the Image Registration Technique from Functional Near Infrared Spectroscopy (fNIRS) Signal and Positron Emission Tomography (PET) Image}

By using $\mathrm{T}_{\mathrm{fNIRS}} \rightarrow$ Pol_1, coordinate of probe, $\mathrm{P}_{\text {Pol_1 }}(\mathrm{x}, \mathrm{y}, \mathrm{z})$ in CPol_1 was calculated by the following equation;

$$
\mathrm{P}_{\text {Pol_1 }}=\mathrm{T}_{\mathrm{fNIRS}} \rightarrow \text { Pol_1 }{ }^{*} \mathrm{P}_{\mathrm{fNIRS}}
$$

The coordinate of marker, $\mathrm{M}_{\text {Pol_1 }}(\mathrm{x}, \mathrm{y}, \mathrm{z})$ in $\mathrm{C}$ Pol_1 is corresponded to $\mathrm{M}_{\text {Pol_2 }}(\mathrm{x}, \mathrm{y}, \mathrm{z})$ given by Polaris in $\mathrm{C}_{\mathrm{Pol} \_2}$, as given by the following relationship;

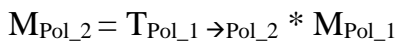

Therefore, TPol_1 $\rightarrow$ Pol_2 is given by the following relationship;

$$
\mathrm{T}_{\mathrm{Pol} \_1} \rightarrow \mathrm{Pol} \_2=\mathrm{M}_{\mathrm{Pol} \_2} * \mathrm{M}_{\mathrm{Pol} \_1}{ }^{-1}
$$

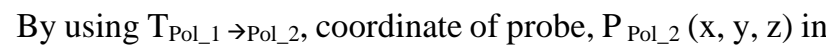
$\mathrm{C}_{\mathrm{Pol} \_2}$ was calculated by the following equation;

$$
\mathrm{P}_{\mathrm{Pol} \_2}=\mathrm{T}_{\mathrm{fNIRS}} \rightarrow \mathrm{Pol} \__{1} * \mathrm{P}_{\mathrm{Pol} \_1}
$$

The coordinate of probe, $\mathrm{P}_{\mathrm{Pol} \_2}(\mathrm{x}, \mathrm{y}, \mathrm{z})$ and marker, $\mathrm{M}_{\text {Pol_2 }}$ $(\mathrm{x}, \mathrm{y}, \mathrm{z})$ in $\mathrm{C}_{\mathrm{Pol} \_2}$ is corresponded to $\mathrm{P}_{\mathrm{PET}}(\mathrm{x}, \mathrm{y}, \mathrm{z})$ and $\mathrm{M}_{\mathrm{PET}}(\mathrm{x}$, $\mathrm{y}, \mathrm{z}$ ) in СРET, as given by the following relationship;

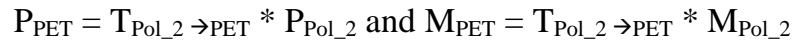

The $\mathrm{T}_{\text {Pol_2 } \rightarrow \text { PET }}$ is given by the calibration experiment using sodium point source $\left({ }^{22} \mathrm{Na}\right)$. By using $\mathrm{T}_{\mathrm{Pol} \_2} \rightarrow$ PET , coordinate of probe, $\mathrm{P}_{\mathrm{PET}}(\mathrm{x}, \mathrm{y}, \mathrm{z})$ in $\mathrm{C}_{\mathrm{PET}}$ was calculated by the following equation;

$$
\mathrm{P}_{\mathrm{PET}}=\mathrm{T}_{\mathrm{Pol} \_2 \rightarrow \mathrm{PET}} * \mathrm{P}_{\mathrm{Pol} \_2}
$$

as well as coordinate of marker coordinate.

$$
\mathrm{M}_{\mathrm{PET}}=\mathrm{T}_{\mathrm{Pol} \_2 \rightarrow \mathrm{PET}} * \mathrm{M}_{\mathrm{pol} \_2}
$$

\section{RESULT}

\section{A. The transformation}

Fig. 8 shows the theory of transformation from fNIRS to PET. The probes and markers position on the phantom were transformed to the Polaris 1 coordinate $\left(\mathrm{C}_{\mathrm{Pol} \_1}\right)$ and Polaris 2 coordinate ( $\mathrm{C}_{\text {Pol_2 }}$ ) before transformed to C СЕT. During fNIRS measurement, the Polaris system read the position of Polaris markers as well as during the PET measurement separately, thus; the $\mathrm{C}_{\mathrm{Pol} \_1}$ is the Polaris coordinate during fNIRS measurement while $\mathrm{C}_{\mathrm{Pol} \_2}$ is Polaris coordinate during PET scan. The transformation error from fNIRS to Polaris and Polaris to CPET is less than $1.00 \mathrm{~mm}$ as presented in table 1 . The highest error is $0.89 \mathrm{~mm}$ at $\mathrm{Y}$ axis from Polaris to PET.

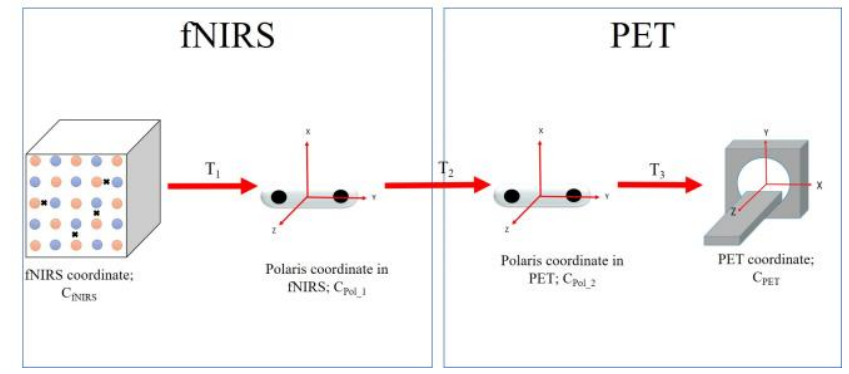

Fig. 8. The illustration of the flow of the transformation done on the phantom. The phantom coordinate in fNIRS was transformed to Polaris coordinate and PET coordinate.
Table I: Transformation error from fNIRS to Polaris and from Polaris to PET

\begin{tabular}{|c|c|c|}
\hline \multirow{2}{*}{ Axis } & \multicolumn{2}{|c|}{ Transformation error (mm) } \\
\cline { 2 - 3 } & From fNIRS to Polaris & From Polaris to PET \\
\hline x-axis & $2.32 \times 10^{-8} \pm 5.88 \times 10^{-9}$ & $0.87 \pm 0.053$ \\
\hline y-axis & $1.65 \times 10^{-6} \pm 2.94 \times 10^{-7}$ & $0.89 \pm 0.058$ \\
\hline z-axis & $1.12 \times 10^{-6} \pm 2.18 \times 10^{-7}$ & $0.32 \pm 0.041$ \\
\hline
\end{tabular}

\section{B. The registration}

The transformation result in the position of the probe and Polaris markers in PET coordinate and the probes was then registered on the phantom PET image using in house software. The illustration of the probes position, Polaris markers and Na-22 source at channel 6 in PET coordinate are presented in fig. 9. The fusion image of the registration result was shown in fig. 10, it clearly seen that the source is located at channel 6 in the fusion image, compare to actual position as illustrated in fig. 9.

\section{Precision and accuracy test}

During fNIRS measurement, the measurement was repeated at different position to test the precision of the transformation. The precision test compared the measurement of phantom at three different positions. This is done by calculating the standard deviation of the differences of the Polaris markers, M1, M2, M3 and M4 coordinate in three different position of the phantom and the result presented in in bar chart form in fig. 11. The precision test shows that the standard deviation is less than $0.1 \mathrm{~mm}$ which mean that the transformation is consistence even though the phantom was moved to different position during fNIRS measurement.

Accuracy test was calculated by comparing the position of the channel 6 in $\mathrm{CPET}_{\text {PE }}$ with the actual position of the source in PET image. Since the position of the source was above the actual position of the phantom, so, only $\mathrm{x}$-axis can be compared. Fig. 12 shows the standard deviation of the three position of the phantom compare to the actual position of channel 6 . The result shows the accuracy is less than $2.8 \mathrm{~mm}$.

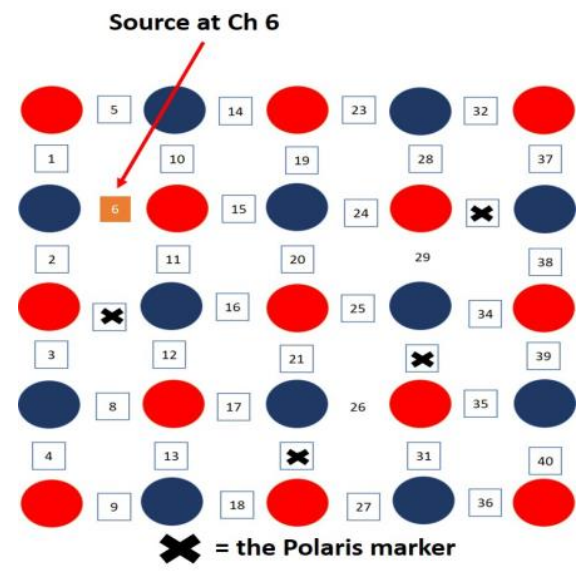

Fig. 9. The illustration of channel 6, the fNIRS probes and the Polaris markers in $\mathrm{C}_{\text {PET. }}$. The blue and red dot are the probes

Published By:

Blue Eyes Intelligence Engineering

\& Sciences Publication

(C) Copyright: All rights reserved.

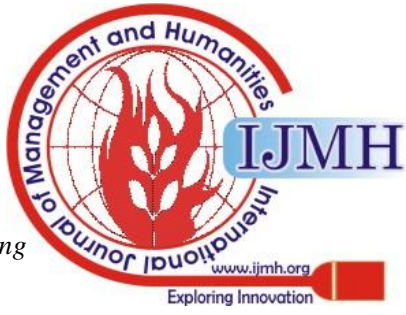




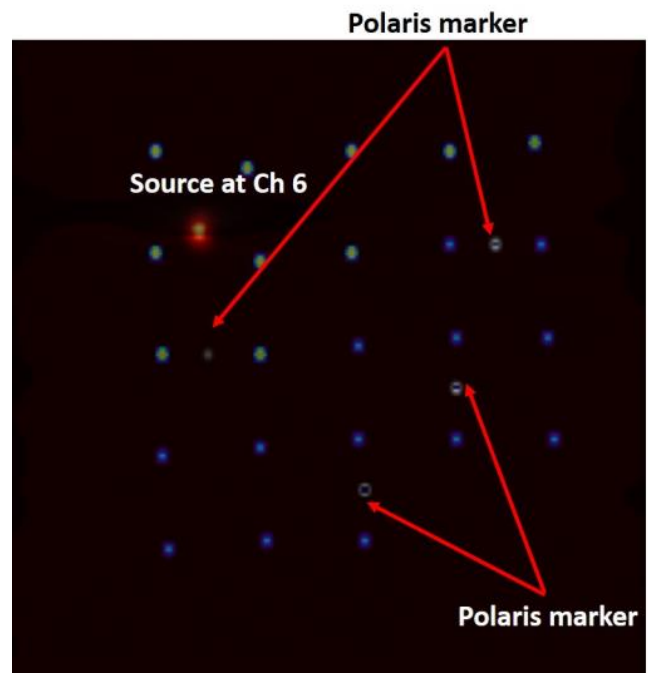

Fig. 10. The fusion image on the source on channel 6, the probe and the Polaris markers image. The blue 'dot' are the probes, the white 'dot' (appointed by the arrows) are the markers while the red ' $\mathrm{dot}^{\prime}$ ' is the source.

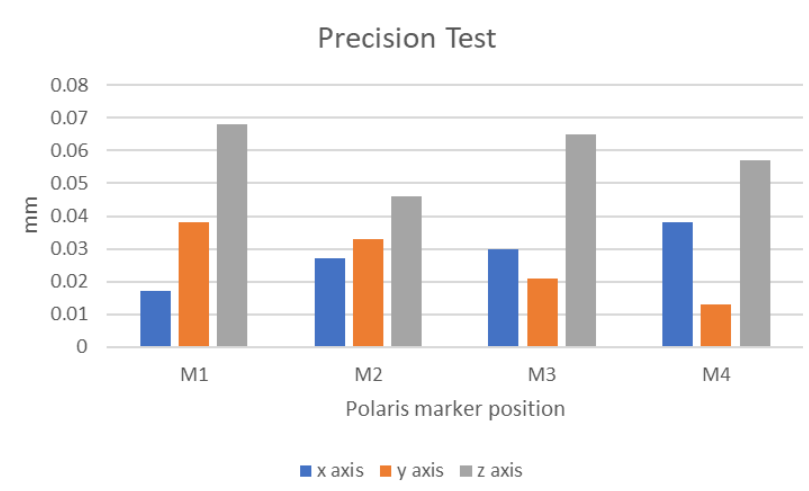

Fig. 11. The precision test of the markers position in three different position.

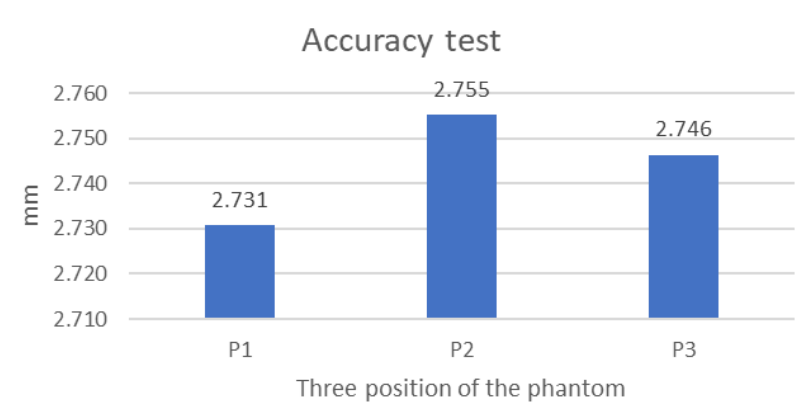

Fig. 12. The accuracy test of the marker coordinate in $\mathrm{x}$-axis at three different position presented in bar chart.

\section{DISCUSSION}

The validation experiment showed that the fNIRS probes and Polaris markers can be observed in the PET image from the fusion images as shown in fig. 12. This method involves several transformations from the fNIRS coordinate to the PET coordinate. Because of this, it is important to identify the transformation error. The transformation error from fNIRS to Polaris and Polaris to PET coordinate is less than $1.00 \mathrm{~mm}$ at each $\mathrm{x}, \mathrm{y}$ and $\mathrm{z}$-axis. The precision test compared the measurement of phantom at three different positions and the differences is less than $0.1 \mathrm{~mm}$ which indicate that the transformation is consistence even though the phantom was moved to different position during repetaed fNIRS measurement. Therefore, this method can be used regardless to where the fNIRS is measured. Meanwhile, the accuracy test compared the position of the source on channel 6 after the transformation to the PET coordinate and the actual position of the source. The accuracy test shows the differences of the actual coordinate of the source and the transformation coordinate from fNIRS is less than $2.8 \mathrm{~mm}$. As the marker diameter is $9 \mathrm{~mm}$, therefore the value $2.8 \mathrm{~mm}$ is within the diameter of the Polaris marker. It is very important to ensure the accuracy of the registration before they are used for the clinical or research application. By registering the fNIRS probe on the PET image, the fNIRS channels is also registered as well so the fNIRS signal can be view on the PET image. This registration technique is hardware-based registration as we used Polaris, the optical tracking system to track the Polaris markers during both procedures. The imaging coordinate of PET and fNIRS are calibrated to each other by Polaris, so the fNIRS is brought into the same physical location of PET and assume that the patient or subject remains motionless between both fNIRS and PET acquisition. The technique of calibrated coordinate systems is also often used in registering the ultrasound to CT or MRI and registering the position of surgical tools mounted on a robot arm to images [12]. The proposed registration methods using hardware tracking system is inexpensive method and very convenient to register fNIRS and other imaging modality. It is possible to apply this method to register fNIRS with other imaging modalities such as MRI, CT scan and SPECT however several considerations should be taken. fNIRS is radiation safe, non-invasive method and inexpensive compare to other brain imaging modalities. Thus, fNIRS could be a better option for multimodal imaging. fNIRS also portable and regardless to motion and it is very convenient to monitor oxygen to the brain area during activities. The change in hemodynamic response is a good marker for assessing neural activity since the oxygenated level is dependent on brain activities and mostly fNIRS measurement is done based on mental work in human [13][14]. Thus, many researchers tend to combine fNIRS with other application especially fMRI. The combination of fNIRS and fMRI studies has been reviewed in Scarapicchia et al., 2017 and there are over 100 published articles using combined fMRI-fNIRS in brain function studies [15]. Hence, it is very useful if the information given by fNIRS can be combined with image from other imaging modalities. Thus, this registration method can be used to view the fNIRS signal on the image from other modalities. fNIRS signal is presented in 2D signals form and fNIRS is obtained from the brain surface without the structural and anatomical information about the underlaying brain. Thus, fNIRS signal is presented in either Montreal Neurological Institute (MNI) or Talairach space [16] or superimposed onto the standard MRI brain template images by fNIRS-SPM in order to view the area of the fNIRS signal on the brain region.

Published By:

Blue Eyes Intelligence Engineering

\& Sciences Publication

(C) Copyright: All rights reserved.

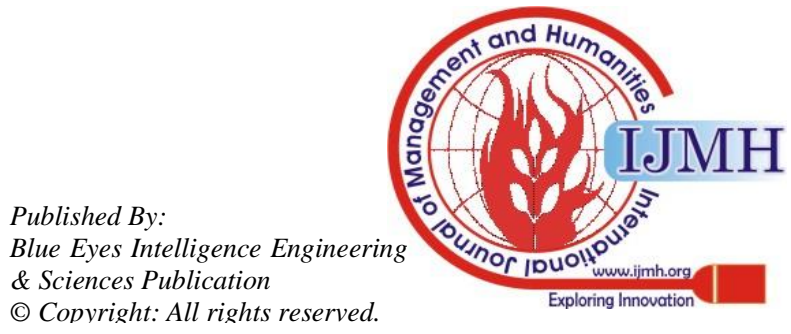




\section{Validation of the Image Registration Technique from Functional Near Infrared Spectroscopy (fNIRS) Signal and Positron Emission Tomography (PET) Image}

In Kikuchi et al., 2018, fNIRS signal is superimposed on standard MRI template images to compare the sedative effects of antihistamines in term of cerebral glucose metabolic changes measured by PET and hemodynamic response measured by fNIRS [17]. Therefore, if fNIRS signal could be view on the own subject image, we can view both information in one image of the same subject. Although fNIRS measures only in the cortex region, it is useful to determine whether the hemodynamic changes that given by fNIRS and metabolic changes observed by PET are occurs in the same brain region or not. fNIRS is measured using probes on the brain scalp. It is important to view the underlying structure of the fNIRS signal. By registering fNIRS on the subject individual brain image, the information gathered will be expanded to the brain structure or clinical abnormalities together with the information given by fNIRS. But to register fNIRS on individual image, it is very complex because every human has their unique shape and size of the brain. Therefore, there are many studies register the fNIRS on the standard MRI image. Tsuzuki \& Dan 2014., described the fNIRS data on the standard stereotaxic space to register the fNIRS probe on the brain MRI image, either on the subject own's MRI image or MRI standard image [16]. Other registration of fNIRS was describe by Okamoto \& Dan 2005, where they developed two transcranial projection algorithms that project given head-surface points onto the cortical surface in structural images; the convex-hull algorithm and the balloon-inflation. This software-based method proposed the automated transcranial projection to register the fNIRS probe on the standard MRI image[18]. Hence in our proposed method, the registration of fNIRS can be done with PET and other modalities as well and it is very convenient for those places without MRI since installation of MRI is very costly.

Our proposed method of registration is very useful to register fNIRS of individual subject on their own PET image and can be applied by researcher. However, there are barrier to the routine clinical use in term of logistical difficulties in getting image to be register one by one. In future, a standard software should be developed for the effectiveness of the registration process.

\section{CONCLUSION}

In this paper we described and validated the registration methods of fNIRS signal and PET image. The precision and accuracy of this method is small, and this method could be used as based to produce fusion image of fNIRS and PET. Using this method, the fNIRS signal at the activation area could be view on the PET image. By registering fNIRS channel on the subject own PET image, we can compare and widen the information given by both modalities. In turn, fNIRS also can be register on image acquire by other imaging modalities and in some ways, multimodal interaction of fNIRS is a good research development of fNIRS in future

\section{ACKNOWLEDGMENT}

This study was supported by Grants-in-Aid for Scientific Research No. 17 H04118 from the Ministry of Education, Culture, Sports, Science and Technology (MEXT), Japanese Government.

\section{REFERENCES}

1. Fitzpatrick JM, Hill DLG, Maurer CR. CHAPTER 8 Image Registration. In: Sonka Mi, Fitzpatrick JMi, editors. Handb. Med. Imaging. volume 2, SPIE-The international Society for Optical Enginnering; 2004, p. 447-514.

2. Gervain J. Near-Infrared Spectroscopy. vol. 16. Second Edi. Elsevier; 2015. https://doi.org/10.5772/32493.

3. Sato H, Yahata N, Funane T, Takizawa R, Katura T, Atsumori H, et al. A NIRS-fMRI investigation of prefrontal cortex activity during a working memory task. Neuroimage 2013;83:158-73. https://doi.org/10.1016/j.neuroimage.2013.06.043.

4. Heinzel S, Haeussinger FB, Hahn T, Ehlis AC, Plichta MM, Fallgatter AJ. Variability of (functional) hemodynamics as measured with simultaneous fNIRS and fMRI during intertemporal choice.Neuroimage 2013;71:125-34. https://doi.org/10.1016/j.neuroimage.2012.12.074.

5. Duan L, Zhang YJ, Zhu CZ. Quantitative comparison of resting-state functional connectivity derived from fNIRS and fMRI: A simultaneous recording study. Neuroimage 2012;60:2008-18. https://doi.org/10.1016/j.neuroimage.2012.02.014.

6. Cui X, Bray S, Bryant DM, Glover GH, Reiss AL. A quantitative comparison of NIRS and fMRI across multiple cognitive tasks. Neuroimage https://doi.org/10.1016/j.neuroimage.2010.10.069.

7. Magnotta VA, Buss AT, Huppert TJ, Spencer JP, Wijeakumar S. Validating an image-based fNIRS approach with fMRI and a working memory task. Neuroimage 2016;147:204-18. https://doi.org/10.1016/j.neuroimage.2016.12.007.

8. Kleinschmidt A, Obrig $\mathrm{H}$, Requardt $\mathrm{M}$, Merboldt $\mathrm{K}$, Dirnagl U, Villringer A, et al. Simultaneous Recording of Cerebral Blood Oxygenation Changes During Human Brain Activation by Magnetic Resonance Imaging and Near-Infrared Spectroscopy 1996:817-26.

9. Rostrup E, Law I, Pott F, Ide K, Knudsen GM. Cerebral hemodynamics measured with simultaneous PET and near-infrared spectroscopy in humans. Brain Res 2002;954:183-93. https://doi.org/10.1016/S0006-8993(02)03246-8.

10. Polinder-Bos HA, Elting JWJ, Aries MJH, García DV, Willemsen ATM, van Laar PJ, et al. Changes in cerebral oxygenation and cerebral blood flow during hemodialysis - A simultaneous near-infrared spectroscopy and positron emission tomography study. J Cereb Blood Flow Metab 2018. https://doi.org/10.1177/0271678X18818652.

11. Mohd Nasir F, Kikuchi A, Miyake M, Watanuki S, Tashiro M, Watabe H. Registration of Positron Emission Tomography (PET) Image and Functional Near Infrared Spectroscopy (NIRS) Data. Int J Biosci Biochem Bioinforma 2019;9:9-19. https://doi.org/10.17706/ijbbb.2019.9.1.9-19

12. Maintz JBA, Viergever $M$ a. An Overview of Medical Image Registration Methods (Cited by: 2654). Nature 1996;12:1-22. https://doi.org/10.1.1.39.4417.

13. Hoshi Y, Tamura M. Detection of dynamic changes in cerebral oxygenation coupled to neuronal function during mental work in man. Neurosci Lett 1993;150:5-8. https://doi.org/10.1016/0304-3940(93)90094-2.

14. Hoshi Y, Tamura M. Dynamic multichannel near-infrared optical imaging of human brain activity. J Appl Physiol 2017;75:1842-6. https://doi.org/10.1152/jappl.1993.75.4.1842.

15. Scarapicchia V, Brown C, Mayo C, Gawryluk JR. Functional Magnetic Resonance Imaging and Functional Near-Infrared Spectroscopy: Insights from Combined Recording Studies. Front Hum Neurosci 2017;11:1-12. https://doi.org/10.3389/fnhum.2017.00419.

16. Tsuzuki D, Dan I. Spatial registration for functional near-infrared spectroscopy: From channel position on the scalp to cortical location in individual and group analyses. Neuroimage 2014;85:92-103. https://doi.org/10.1016/j.neuroimage.2013.07.025.

17. Kikuchi A, Nasir FMN, Inami A, Mohsen A, Watanuki S, Miyake M, et al. Effects of levocetirizine and diphenhydramine on regional glucose metabolic changes and hemodynamic responses in the human prefrontal cortex during cognitive tasks. Hum Psychopharmacol 2018;33:1-16. https://doi.org/10.1002/hup.2655.

18. Okamoto M, Dan I. Automated cortical projection of head-surface locations for transcranial functional brain mapping. Neuroimage 2005;26:18-28. https://doi.org/10.1016/j.neuroimage.2005.01.018. 


\section{AUTHORS PROFILE}

Fairuz binti Mohd Nasir, was born in Kedah, Malaysia. She graduated MSc in Medical Physics and BSc in Medical Radiation from University Sains Malaysia. She works as lecturer at Faculty of Health Science, in University Sultan Zainal Abidin, Malaysia from 2010 till present. She holds a license of Radiation Protection Officer since 2007 and was appointed as Radiation Protection Officer since 2007 till 2015. Currently, she is a PhD student in the Graduate School of Biomedical Engineering, Tohoku University, Japan. Her current study is about registering the NIRS and PET. She has presented her works during Japanese Society of Nuclear Medicine 2016 conference in Nagoya, Japan. Her research interest is radiation physics, image processing, and radiation protection.

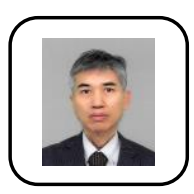

Hiroshi Watabe, was born in Shizuoka, Japan in 1967. He was received Ph.D. in nuclear engineering from Tohoku University, Sendai, Japan in 1995. During his Ph.D. course, he was at Medical Research Council Cyclotron Unit, Hammersmith Hospital, London, UK in 1993 and 1994. In 1995-2009, he was at National Cardiovascular Center Research Institute, Osaka, Japan as a research staff and a section chief. In 1997-1999, he spent as a visiting fellow in PET Department, National Institutes of Health, Bethesda, U.S.A. From 2009 to 2013, he was an associate professor of Department of Molecular Imaging in Medicine, Osaka University Graduate School of Medicine, Osaka, Japan. Since 2013, he has been in Division of Radiation Protection \& Safety Control, Cyclotron and Radioisotope Center, Tohoku University, Sendai, Japan. Since 2015, he has been a professor. His research interest includes nuclear medicine physics, nuclear engineering in medicine and radiation dosimetry.

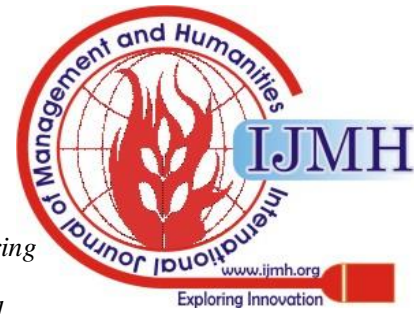

